\title{
Apnea-Induced Rapid Eye Movement Sleep Disruption Impairs Human Spatial Navigational Memory
}

\author{
Andrew W. Varga, ${ }^{1,3}$ Akifumi Kishi, ${ }^{1}$ Janna Mantua, ${ }^{1}$ Jason Lim, ${ }^{1}$ Viachaslau Koushyk, ${ }^{1}$ David P. Leibert, ${ }^{1}$ \\ Ricardo S. Osorio, ${ }^{2}$ David M. Rapoport, ${ }^{1}$ and Indu Ayappa ${ }^{1}$ \\ ${ }^{1}$ New York University (NYU) Sleep Disorders Center and ${ }^{2}$ Center for Brain Health, NYU Langone School of Medicine, NYU, New York, New York 10016, and \\ ${ }^{3}$ Center for Neural Science, New York University, New York, New York 10003
}

\begin{abstract}
Hippocampal electrophysiology and behavioral evidence support a role for sleep in spatial navigational memory, but the role of particular sleep stages is less clear. Although rodent models suggest the importance of rapid eye movement (REM) sleep in spatial navigational memory, a similar role for REM sleep has never been examined in humans. We recruited subjects with severe obstructive sleep apnea (OSA) who were well treated and adherent with continuous positive airway pressure (CPAP). Restricting CPAP withdrawal to REM through real-time monitoring of the polysomnogram provides a novel way of addressing the role of REM sleep in spatial navigational memory with a physiologically relevant stimulus. Individuals spent two different nights in the laboratory, during which subjects performed timed trials before and after sleep on one of two unique 3D spatial mazes. One night of sleep was normally consolidated with use of therapeutic CPAP throughout, whereas on the other night, CPAP was reduced only in REM sleep, allowing REM OSA to recur. REM disruption via this method caused REM sleep reduction and significantly fragmented any remaining REM sleep without affecting total sleep time, sleep efficiency, or slow-wave sleep. We observed improvements in maze performance after a night of normal sleep that were significantly attenuated after a night of REM disruption without changes in psychomotor vigilance. Furthermore, the improvement in maze completion time significantly positively correlated with the mean REM run duration across both sleep conditions. In conclusion, we demonstrate a novel role for REM sleep in human memory formation and highlight a significant cognitive consequence of OSA.
\end{abstract}

Key words: continuous positive airway pressure (CPAP); obstructive sleep apnea; randomized controlled trial; REM sleep; sleep fragmentation; spatial navigational memory

\section{Introduction}

Both sleep duration and architecture have been implicated in different types of memories, but the mechanisms by which sleep

Received Aug. 4, 2014; revised Sept. 12, 2014; accepted Sept. 18, 2014.

Author contributions: A.W.V., D.M.R., and I.A. designed research; A.W.V., J.M., J.L., and V.K. performed research; A.K., D.P.L., and R.S.O. contributed unpublished reagents/analytic tools; A.W.V., A.K., and D.P.L. analyzed data; A.W.V., D.M.R., and I.A. wrote the paper.

This work was supported by the philanthropy of the James Kuhn Friends of Sleep Medicine, New York University Clinical and Translational Science Awards Grant UL1TR000038 from the National Center for the Advancement of Translational Science (A.W.V.), the American Sleep Medicine Foundation Physician Scientist Training Award (A.W.V.), National Institute of Environmental Health Sciences Training Grant T32ES007267-20 (Principal Investigator William N. Rom), and National Heart, Lung, and Blood Institute K24 Grant HL109156 (I.A.). We thank Dr. Omar Burschtin for experimental insight and critical appraisal of this manuscript and Rakhil Kanevskaya and Boris Opancha for expert sleep scoring. We thank Drs. Robert Stickgold, Erin Wamsley, and Matthew Tucker for virtual maze template and performance analysis guidance.

I.A. has received support for research from the industry for the past 24 months in the forms of grants and clinical trials from Fisher \& Paykel Healthcare. She holds multiple U.S. and foreign patents covering techniques and analysis algorithms for the diagnosis of obstructive sleep apnea/hypopnea syndrome (OSAHS) and techniques for administering continuous positive airway pressure (CPAP). Several of these have been licensed to Fisher \& Paykel Healthcare. D.M.R. has received support for research from the industry for the past 24 months in the form of grants and clinical trials from Fisher \& Paykel Healthcare and speaking and consulting engagements for Fisher \& Paykel Healthcare. He holds multiple U.S. and foreign patents covering techniques and analysis algorithms for the diagnosis of OSAHS and techniques for administering CPAP. Several of these have been licensed to Biologics, Fisher \& Paykel Healthcare, and Health C'Aire.

Correspondence should be addressed to Andrew W. Varga, New York University Sleep Disorders Center, NBV-7N2, 462 First Avenue, New York, NY 10016. E-mail: andrew.varga@nyumc.org.

A. Kiski's present address: Graduate School of Education, University of Tokyo, 7-3-1 Hongo, Bunkyo-ku, Tokyo 113-0033, Japan. exerts these actions remain poorly understood (Diekelmann and Born, 2010). Rapid eye movement (REM) and non-REM (NREM) sleep differ in several significant ways, including neurotransmitter tone, degree of neuronal synchrony, and frequency of cortical local field potential oscillations. Understanding the contributions of REM and NREM sleep toward memory processes may help shed light on the underlying mechanisms.

Spatial navigational memory represents an attractive model memory system to study the effects of sleep. In vivo electrophysiological recordings in actively exploring rodents showed that patterns of firing in hippocampal place cells during wake exploration were replayed during sleep, raising the possibility that this phenomenon acted to strengthen the memory trace. Hippocampal replay occurred in both NREM sleep (Wilson and McNaughton, 1994), in which the hippocampal replay happened over timescales much faster than occurred during wake, and in REM sleep (Louie and Wilson, 2001), in which the replay happened with greater temporal fidelity to wake. Behaviorally, hippocampal sharp-wave ripple complex suppression during NREM sleep impaired performance on a radial arm maze (Girardeau et al., 2009). REM sleep suppression impaired spatial memory in the

DOI:10.1523/JNEUROSCI.3220-14.2014

Copyright $\odot 2014$ the authors $\quad 0270-6474 / 14 / 3414571-07 \$ 15.00 / 0$ 
radial arm maze (Smith et al., 1998) and in the Morris water maze (Youngblood et al., 1997).

Contributions of REM and NREM sleep toward spatial memory in human subjects are less well delineated. Overnight sleep in general enhanced spatial navigational memory as assessed via performance on a virtual maze navigation task that was not appreciated after a comparable period of daytime wake in human subjects (Ferrara et al., 2008; Nguyen et al., 2013). In six subjects, the degree of regional cerebral blood flow in the right hippocampus during slow-wave sleep correlated with overnight performance improvement in a virtual spatial maze (Peigneux et al., 2004). The potential contribution of REM sleep toward consolidation of human spatial navigational memories has heretofore not been examined.

Such a contribution of REM sleep has important clinical implications in the most common medical sleep disorder, obstructive sleep apnea (OSA). Because of the skeletal muscle atonia that normally occurs in REM sleep, the frequency of obstructive apneas and the drop in blood oxygen saturation is often greatest in REM sleep. Few subjects have exclusively REM-related OSA, although many with OSA have their worst disease in REM.

The present study was designed to circumvent this limitation in subject availability. To test the effects of REM-only OSA, we recruited subjects with severe underlying OSA who were well treated and highly adherent to therapy with continuous positive airway pressure (CPAP). Transiently discontinuing therapy for OSA by acutely lowering CPAP has caused immediate recurrence of OSA and many of its consequences (Kohler et al., 2011). Restricting this discontinuation of therapy to a specific stage of sleep (e.g., REM) through real-time overnight EEG monitoring provides a unique and novel way of addressing the role of REM in memory in a human model.

\section{Materials and Methods}

Participants. Subjects were recruited from the New York University (NYU) Sleep Disorders Center. Twenty-one adult subjects with previously diagnosed severe OSA who had been on CPAP therapy for at least 2 months and reported good adherence to CPAP were approached for participation in the study. Severe OSA was defined as having an apneahypopnea index with $4 \%$ oxygen desaturation (AHI4\%) $>30 / \mathrm{h}$ or apnea-hypopnea index with $3 \%$ oxygen saturation or arousal (AHI-all) $>45 / \mathrm{h}$ (see definitions under Polysomnography below). Subjects were included only if CPAP adherence was $\geq 4$ h usage per night for $\geq 70 \%$ of nights as documented by download from the CPAP memory card. Exclusion criteria included use of any psychoactive drugs aside from the use of a single selective serotonin reuptake inhibitor (SSRI) antidepressant, the presence of critical comorbid conditions, the presence of preexisting cognitive or neurological deficits, and pregnancy. One subject was excluded when his CPAP machine data download indicated he had not been using CPAP at all before the first visit. Two other subjects were excluded because their prescribed CPAP was not therapeutic and left them with significant residual apnea during sleep. The number of subjects thus analyzed was 18 ( 14 men, 4 women; mean age, 54 years). Three of the 18 were taking an SSRI antidepressant. All subjects provided informed consent before their participation. All procedures were approved by the NYU Langone School of Medicine Institutional Review Board.

Virtual maze performance and psychomotor vigilance test. At $\sim 8: 00$ P.M. on each night of study, subjects began training on a virtual maze task: a simple 3D environment designed for this research (Nguyen et al., 2013). After a period of generalized familiarization with joystick controls (Thrustmaster) in a Z-shaped corridor, subjects initially spent $3 \mathrm{~min}$ exploring a complex maze designed using "Unreal Tournamant 3 Editor" (Epic Games). Avatar walking speed and turning speed were reduced to minimize motion "cybersickness." The game was projected onto a screen in a darkened testing room. The viewing area was $67 \times 50$ inches, and subjects sat 13 feet away. Subjects were instructed to remember the layout of the maze environment as well as possible. Subsequently, subjects navigated through the same maze during three pre-sleep test trials, in which they were instructed to reach a specified goal point as quickly as possible. Time to reach the goal per trial was capped at $600 \mathrm{~s}$.

Subjects were connected to polysomnography equipment at $\sim 9: 00$ P.M., and a full night polysomnogram on therapeutic CPAP was performed following standard American Academy of Sleep Medicine (AASM) criteria (Iber et al., 2007). On the REM-disruption nights, a sleep technician lowered CPAP to $4 \mathrm{~cm} \mathrm{H}_{2} \mathrm{O}$ as soon as REM sleep was identified and returned to the therapeutic value after exiting REM sleep. During instances in which additional CPAP withdrawal was necessary to induce apnea, CPAP was reduced to $0 \mathrm{~cm} \mathrm{H}_{2} \mathrm{O}$ using equipment and techniques we developed previously to deliver CPAP of $0 \mathrm{~cm} \mathrm{H}_{2} \mathrm{O}$ without rebreathing (Patel et al., 2011).

To control for attention effects, subjects performed a standardized 20 min psychomotor vigilance test (PVT) beginning $1 \mathrm{~h}$ after awakening from each night in the sleep laboratory. Thereafter, subjects performed three final test trials on the $3 \mathrm{D}$ virtual maze, again instructed to reach the same specified goal point as quickly as possible.

Performance metrics on the virtual maze included completion time, distance traveled within the maze, and distance spent backtracking. Overnight change was calculated as the difference between the averages across the three trials before and after sleep, normalized to the average of the three trials before sleep for each metric. Positive values represent improvement, and negative values represent worsening. Performance metrics on the PVT included reaction time (in milliseconds) and number of lapses (no response after $500 \mathrm{~ms}$ ). A transform of the number of lapses was done to enable parametric testing [lapses transform $=$ sqrt (\#lapses) + sqrt (\#lapses + 1), where sqrt is square root] (Dinges et al., 1997).

Subjects were counterbalanced for both maze encountered first and sleep condition encountered first in a pseudorandom manner. A 2 week period occurred between conditions, during which any effect of REM sleep fragmentation, including possible REM rebound, should have normalized if REM disruption occurred first. Subjects returned to the laboratory and performed the exact same series of events under the alternate condition. CPAP adherence was evaluated during the period between conditions. All subjects included in our analysis continued to use CPAP for at least $4 \mathrm{~h} /$ night for at least $70 \%$ of nights (the initial inclusion criterion).

Polysomnography. All polysomnograms consisted of a full night spent in the NYU Sleep Disorders Center. Respiratory airflow was measured from the CPAP machine. CPAP pressure at the mask was measured using a pressure transducer. Polysomnograms were scored in $30 \mathrm{~s}$ epochs according to standard criteria (AASM) for sleep and EEG arousals. Total sleep time and percentage time spent in wake, NREM stage 1 (NREM 1), NREM 2, NREM 3, and REM sleep were determined. Respiratory events were scored from the airflow signal using AASM criteria, and stage specific (REM, NREM, and Total) apnea indices were calculated. Apneas were defined as the absence of airflow for $\geq 10$ s. Hypopnea $4 \%$ was defined as a reduction in the amplitude of breathing by $\geq 30 \%$ for $\geq 10 \mathrm{~s}$ with $\geq 4 \%$ decline in blood oxygen saturation, regardless of the presence of an arousal. Hypopnea ( $3 \%$ or arousal) was defined as a reduction in the amplitude of breathing by $\geq 30 \%$ for $\geq 10$ s accompanied by $\geq 3 \%$ decline in blood oxygen saturation or an arousal indicated by a sudden increase in the cortical EEG frequency or by sudden increase in the motor tone in the chin or anterior tibealis by EMG.

$\mathrm{AHI} 4 \%$ is defined as the sum of all apneas and hypopneas $4 \%$ divided by the total sleep time in hours. AHI-all is defined as the sum of apneas and hypopneas (3\% or arousal) divided by the total sleep time in hours.

Continuity of sleep was assessed as duration of sleep runs, defined as the duration of consecutive $30 \mathrm{~s}$ epochs of sleep scored as that stage, terminated by one or more epochs scored as another stage, including wake (Norman et al., 2006; Kishi et al., 2011).

Data analysis. Data were analyzed using SigmaPlot version 11.0 and MATLAB (R 2013b). Comparison between conditions was performed using paired $t$ tests for normally distributed data, and mean values \pm SEM are reported. For data not normally distributed, analysis consisted of Wilcoxon's signed-rank tests, and median values are reported. 
Table 1. Effects of REM-specific CPAP withdrawal on sleep architecture

\begin{tabular}{lcc}
\hline Sleep measure & Normal sleep on CPAP & REM disrupted \\
\hline Total sleep time & $366.4 \pm 18 \mathrm{~min}$ & $380.0 \pm 16 \mathrm{~min}$ \\
Sleep efficiency & $81.4 \pm 3.3 \%$ & $82.6 \pm 2.0 \%$ \\
\% NREM 1 & $23.1 \pm 2.4 \%$ & $29.5 \pm 2.6 \%^{* *}$ \\
\% NREM 2 & $39.7 \pm 1.8 \%$ & $42.4 \pm 1.7 \%^{*}$ \\
\% NREM 3 & $16.7 \pm 2.3 \%$ & $16.6 \pm 2.6 \%$ \\
\% REM sleep & $20.5 \pm 1.3 \%$ & $11.5 \pm 1.4 \%^{* *}$ \\
\# Arousals in REM & 8.0 (median) & 19.0 (median)** \\
\% REM runs <3 min & $12.2 \pm 2.2 \%$ & $67.6 \pm 5.6 \%^{* *}$ \\
\% REM runs $>5$ min & $79.4 \pm 3.3 \%$ & $19.3 \pm 4.8 \%^{* *}$ \\
Mean REM run duration & $7.44 \pm 0.9$ min & $1.84 \pm 0.2$ min $^{* *}$ \\
\hline
\end{tabular}

Key: NREM1 $=$ non-REM stage 1,NREM2 $=$ non-REM stage 2, NREM3 $=$ non-REM stage $3 .{ }^{*}=p<0.05,{ }^{* *}=p<0.001$.

A

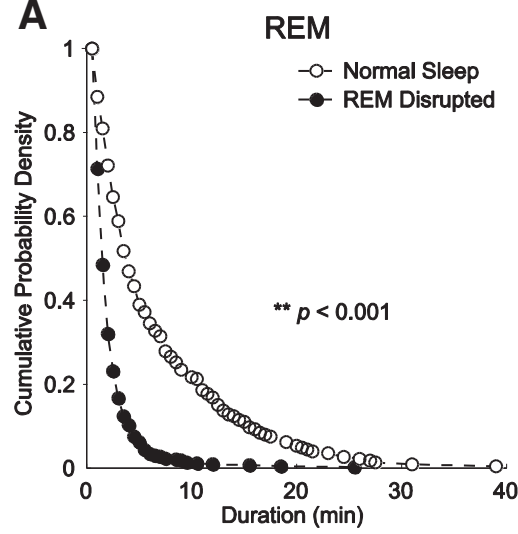

C

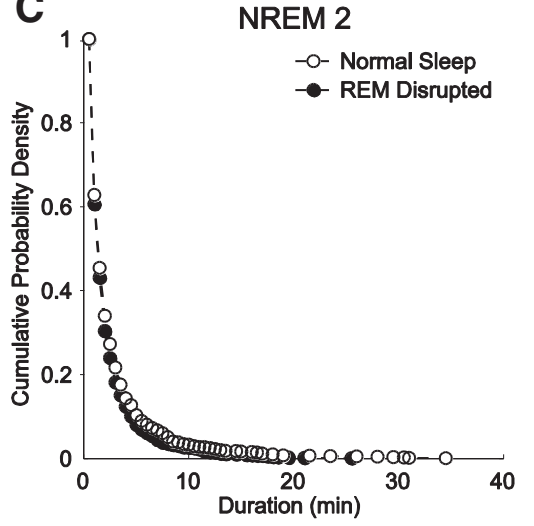

B

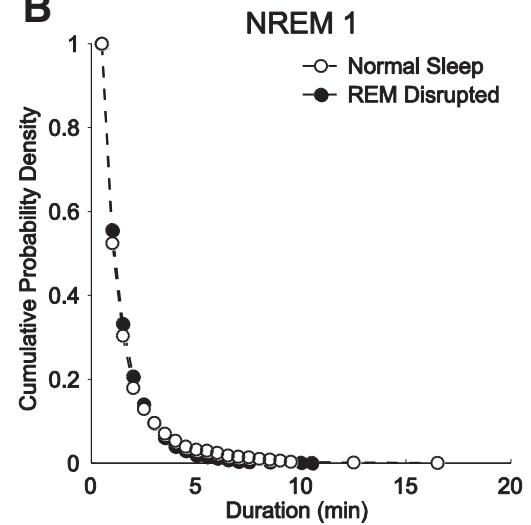

D

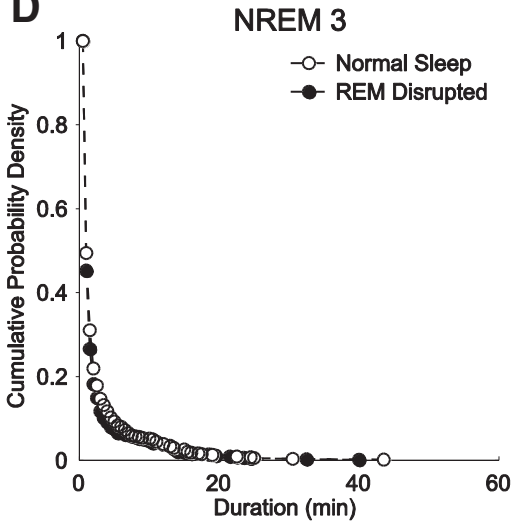

Figure 1. Withdrawal of CPAP in REM sleep causes significant REM sleep fragmentation. Continuity of sleep for normal sleep and REM-disrupted conditions. Survival curves (cumulative probability distributions) of stage-specific sleep runs in the normal sleep (white) and REM-disrupted (black) conditions. $\boldsymbol{A}$, The survival curve for the REM-disrupted night showed a significant shift toward shorter runs of REM sleep compared with the normal night ( $p<0.001$, log-rank test). No significant difference between sleep conditions was observed in the survival curves for NREM 1 (B), NREM 2 (C), or NREM 3 (D).

Table 2. Effects of REM-specific CPAP withdrawal on apnea severity

\begin{tabular}{lcc}
\hline Sleep measure & Normal sleep on CPAP & REM disrupted \\
\hline NREM AHI4\% & $0.65 / \mathrm{h}$ (median) & $3.9 / \mathrm{h}$ (median) \\
NREM AHI-all & $5.7 / \mathrm{h}$ (median) & $12.4 / \mathrm{h}$ (median) \\
REM AHI4\% & $1.2 \pm 0.4 / \mathrm{h}$ & $29.9 \pm 4.2 / \mathrm{h}^{* *}$ \\
REM AHI-all & $9.3 \pm 1.0 / \mathrm{h}$ & $46.1 \pm 3.5 / \mathrm{h}^{* *}$ \\
Total AHI4\% & $1.7 \pm 0.8 / \mathrm{h}$ & $8.1 \pm 1.3 / \mathrm{h}^{* *}$ \\
Total AHI-all & $7.2 \pm 1.4 / \mathrm{h}$ & $17.0 \pm 1.6 / \mathrm{h}^{* *}$ \\
\hline
\end{tabular}

${ }^{*} p<0.05,{ }^{* *} p<0.001$.

For sleep continuity data, a bootstrap-based analysis that accounted for the number of runs contributed by each subject was performed for continuity of each sleep stage (REM, NREM 1, NREM 2, and NREM 3). Nights in which CPAP was withdrawn were likely to result in a significantly higher number of sleep runs versus nights without CPAP withdrawal. Thus, striking a balance between equivalence among subjects and retaining a sufficient number of data points, on those nights with more than the median number of runs, runs were randomly sampled up to the median number. Log rank tests were used to compare REM-disrupted versus normal sleep conditions on the survival curves derived from the sampling procedure. This procedure was repeated 1000 times. At each step of the iteration, a $p$ value was estimated from the history of previous $p$ values, yielding an increasingly stable result as the number of iterations increases. Correlation data were calculated using a Pearson's product moment with mean REM run duration shown on a log scale. Results were considered significant at $p<0.05$.

\section{Results}

Effects of CPAP withdrawal in REM sleep on sleep architecture and OSA severity

Total sleep time and sleep efficiency were markedly similar between conditions. When REM was disrupted via CPAP withdrawal, there were numerically small, but statistically significant, increases in NREM 1 and NREM 2, without any significant change in NREM 3 (slowwave sleep). In contrast, when REM was disrupted via CPAP withdrawal, there was a significant reduction in the proportion of REM sleep from 20.5 to $11.5 \%$. CPAP withdrawal in REM sleep also resulted in significant fragmentation of any remaining REM sleep. The mean REM run duration was significantly deceased in the REM-disrupted condition. The percentage of time spent with runs of REM $<3$ min in duration was significantly increased, and the percentage of time spent with runs of REM $>5$ min was significantly decreased in subjects when CPAP was withdrawn in REM versus normal sleep. The median number of arousals in REM increased when CPAP was withdrawn in REM. Table 1 summarizes the changes in sleep architecture across conditions. The cumulative duration probability distribution of REM sleep during CPAP withdrawal in REM was significantly left shifted $(p<0.001)$, indicating that REM sleep occurred in smaller bouts than during normal sleep, without any significant change in the duration probability distribution of NREM stages (Fig. 1). In summary, CPAP withdrawal in REM sleep induced decreased amounts of REM sleep and produced high levels of fragmentation of the remaining REM sleep.

CPAP withdrawal in REM resulted in small but statistically significant increases in the NREM AHI $4 \%$ and AHI-all, although all values remained in the normal range, suggesting absence of clinical disease $(\mathrm{AHI} 4 \%<5 / \mathrm{h}$; AHI-all $<15 / \mathrm{h})$. As expected, CPAP withdrawal in REM sleep resulted in large significant increases in the $\mathrm{AHI} \% \%$ and $\mathrm{AHI}$-all during REM to ranges consistent with severe disease (AHI $\%>30 / \mathrm{h}$ or AHI-all $>45 / \mathrm{h}$ ). Averaged over the entire night, CPAP withdrawal in REM sleep produced only mild OSA overall $(\mathrm{AHI} 4 \%=8.1 / \mathrm{h}$ and $\mathrm{AHI}-\mathrm{all}=$ $17.0 / \mathrm{h}$ ). Table 2 summarizes the changes in OSA severity. 
A

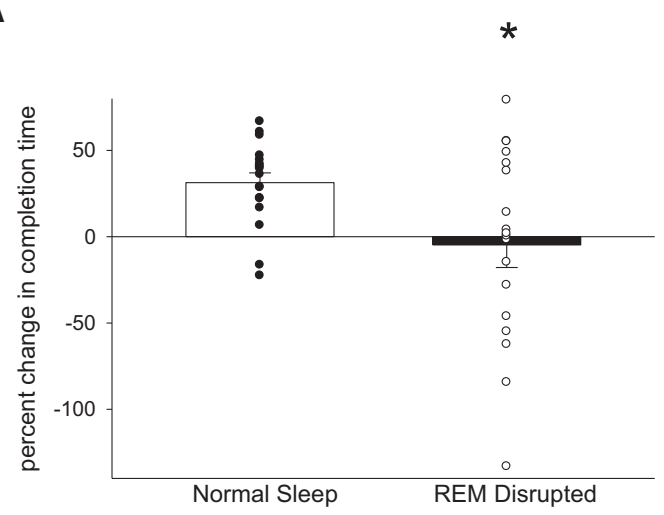

C

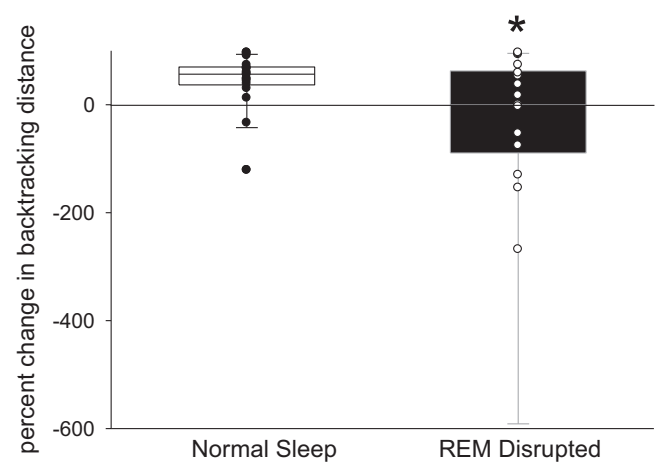

B

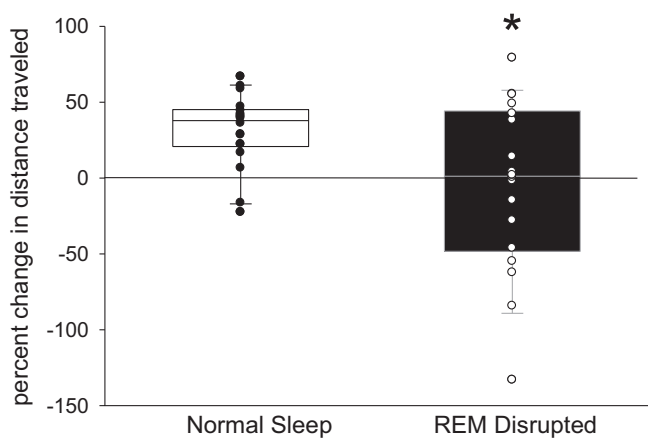

Figure 2. Disruption of REM sleep significantly attenuates the gains in virtual maze performance that occur across normal sleep. $A$, Completion time improved $31.3 \%$ after normal sleep but worsened $4.7 \%$ after REM disruption. ${ }^{*} p=0.016$, paired $t$ test. $\boldsymbol{B}$, Total distance traveled improved $34.3 \%$ after normal sleep but worsened $0.5 \%$ after REM disruption. ${ }^{*} p=0.03$, Wilcoxon's signed-rank test. C, Distance spent backtracking improved $56.5 \%$ after normal sleep but worsened $0.1 \%$ after REM disruption. ${ }^{*} p=0.034$, Wilcoxon's signed-rank test.

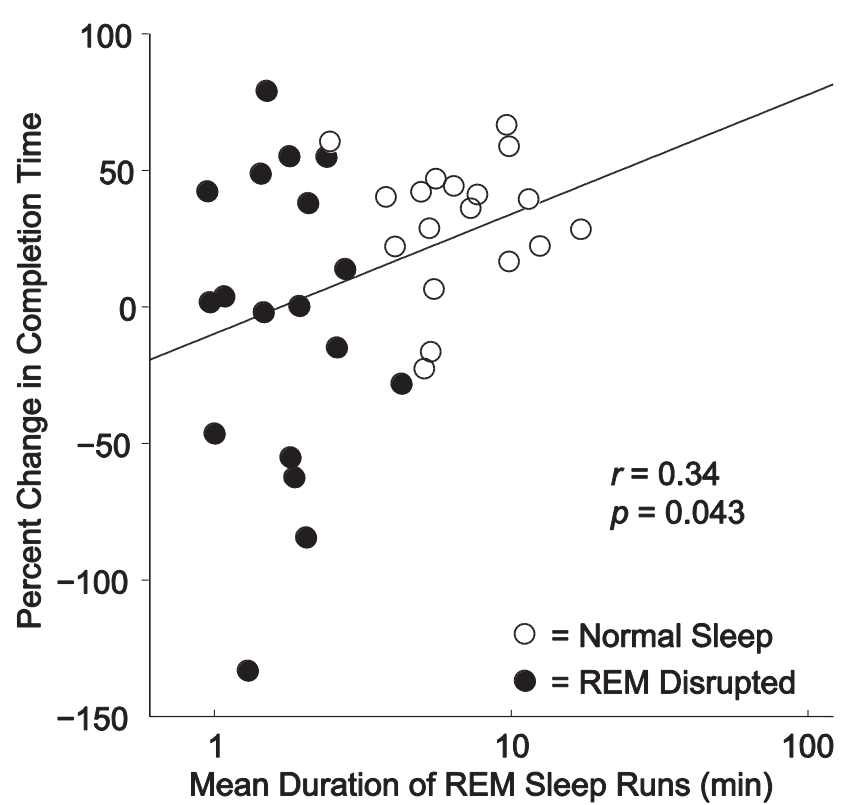

Figure 3. Overnight change in virtual maze completion time positively correlates with the mean REM run duration. Scatter plots of the relationship between REM continuity (mean duration of REM sleep runs) and percentage change in completion time for normal sleep (white circles) and REMdisrupted night (black circles). $r=0.34, p=0.043$, Pearson's correlation coefficient.

\section{Effects of CPAP withdrawal in REM sleep on spatial} navigational memory

We tracked subjects' average performance before and after sleep by measures of completion time, distance traveled within the maze, and distance spent backtracking. Across a night of normal sleep, there was improvement in completion time (mean of $31.3 \%$ ), distance traveled (median of $34.3 \%$ ), and distance spent backtracking (median of 56.5\%). During conditions of REM disruption via CPAP withdrawal, these performance gains were not only lost, but on average, subjects worsened on all three measures: completion time (mean of $-4.7 \%, p=0.016$ vs normal sleep, paired $t$ test), distance traveled (median of $-0.5 \%, p=0.03$ vs normal sleep, Wilcoxon's signed-rank test), distance spent backtracking (median of $-0.1 \%, p=0.034$ vs normal sleep, Wilcoxon's signed-rank test; Fig. 2). Importantly, across all study nights regardless of sleep condition, the degree of change in completion time performance significantly positively correlated with the mean REM run duration $(r=0.34, p=0.043$, Pearson's correlation; Fig. 3) but not with amounts of REM sleep, either by total minutes of REM or percentage of REM sleep or by severity of the REM OSA (AHI4\% or AHI-all). This suggests then that the consolidation of REM sleep is more important than strict duration for optimizing spatial navigational memory.

Although improved performance could have occurred on the second night as a result of enhanced familiarity with the task, we did not observe any significant differences in subjects' performance between night 1 and night 2 regardless of maze or condition (mean completion time improvement night $1=14.4 \%$, night $2=12.1 \%, p=0.89$, paired $t$ test) or between maze 1 and maze 2 regardless of night or condition (mean completion time improvement maze $1=18 \%$, maze $2=8.6 \%, p=0.44$, paired $t$ test). 
A

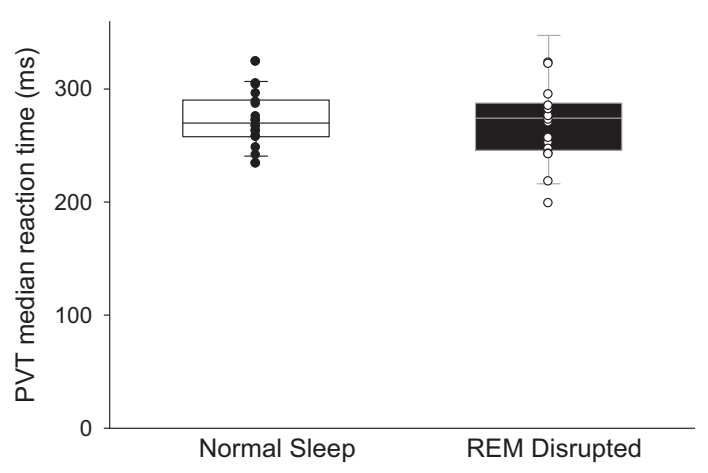

B

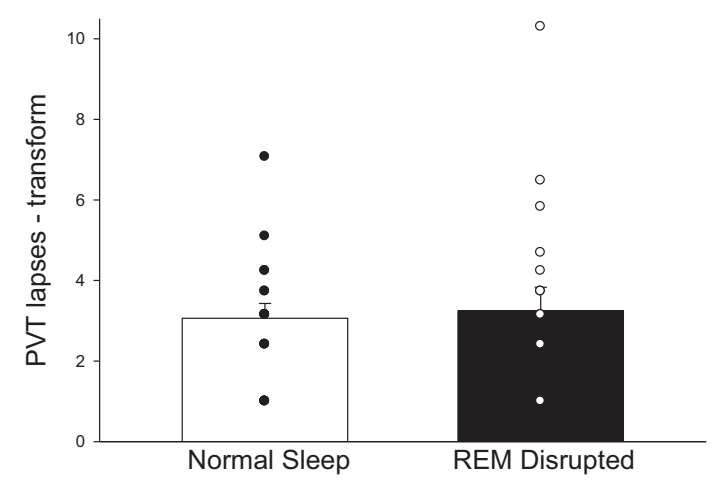

Figure 4. Disruption of REM sleep does not affect psychomotor vigilance. There is no difference in mean reaction time between sleep conditions (270 ms during normal sleep, 274 ms during REM disruption, $p=0.865$, Wilcoxon's signed-rank test) $(\boldsymbol{A})$ or in a transform of lapses (3.1 \pm 0.4 during normal sleep, $3.3 \pm 0.6$ during REM disruption, $p=0.71$, paired $t$ test) $(\boldsymbol{B})$.

\section{Effects of CPAP withdrawal in REM sleep on psychomotor vigilance}

To control for possible effects on attention affecting spatial navigation performance in the morning, subjects performed a 20 min PVT immediately before completing the three morning test trials on the virtual maze. We observed no significant differences in mean reaction times $(270 \mathrm{~ms}$ during normal sleep, 274 ms during REM disruption, $p=0.865$, Wilcoxon's signed rank test) or in number of lapses $(3.1 \pm 0.4$ during normal sleep, $3.3 \pm 0.6$ during REM disruption, $p=0.71$, paired $t$ test; Fig. 4 ).

\section{Discussion}

In this study, we demonstrate that overnight sleep acts to consolidate spatial navigational memories. This benefit of overnight sleep is lost when REM sleep is disrupted by OSA induced by CPAP withdrawal exclusively in REM sleep. Our finding of the $31.3 \%$ overnight improvement in completion time on the virtual maze in our older population was quite similar to the $34 \%$ overnight improvement (Nguyen et al., 2013) and 30\% overnight improvement (Ferrara et al., 2008) in completion time found in healthy cohorts of college-aged subjects on related virtual mazes. That these improvements in completion time, as well as improvements in distance traveled and distance spent backtracking, were ablated by REM disruption suggests that REM sleep is necessary for the consolidation of spatial navigational memory. An alternative interpretation is that the deficit in spatial navigational memory consolidation is a nonspecific consequence of sleep disruption in general, regardless of sleep stage. Such an interpretation supposes that an equivalent amount of sleep disruption (e.g., $11.5 \%$ of total sleep time) occurring randomly across sleep would cause the same deficit. However, previous work has shown that disruption of NREM sleep significantly reduces psychomotor vigilance (Kribbs et al., 1993), thereby potentially confounding the interpretation of worsened virtual maze performance after such an intervention. Importantly, the disruption of REM sleep in this study caused no change in morning psychomotor vigilance.

The induction of OSA in REM sleep produced in our study shortened and fragmented REM sleep but also induced intermittent hypoxia. Although we cannot rule out the contribution of intermittent hypoxia toward interrupting spatial navigational memory consolidation, intermittent hypoxia in rodent models induced deficits in spatial memory in the Morris water maze under chronic, rather than acute, conditions (Gozal et al., 2001). Additionally, in a direct comparison of acute intermittent hypoxia versus sleep fragmentation $24 \mathrm{~h}$ after acquisition of the Morris water maze in rodents, subsequent spatial memory was preserved after intermittent hypoxia but significantly worsened after sleep fragmentation (Ward et al., 2009).

Spatial navigational memory is thought to be subserved by at least two brain systems working in concert: (1) an allocentric system mediated primarily by medial temporal lobe structures in which external landmarks are used to create a mental spatial map; and (2) an egocentric system mediated primarily by the striatum, which incorporates body movement, turn direction, speed, and elapsed time (Iaria et al., 2003; Buzsáki and Moser, 2013). Rodents deprived chronically of either total sleep (Hagewoud et al., 2010) or REM sleep (Bjorness et al., 2005) showed significant inability to engage hippocampal strategies for solving spatial tasks and instead relied on nonspatial, response-based strategies. One night of total sleep deprivation in human subjects resulted in decreased recruitment of bilateral posterior hippocampal regions during encoding of visual scenes measured with functional imaging (Yoo et al., 2007). In contrast, when sleep was completely deprived overnight in human subjects after evening training on a virtual maze, functional imaging during navigation $72 \mathrm{~h}$ later showed absence of recruitment of the caudate bilaterally (Orban et al., 2006).

Our finding of the importance of REM sleep in spatial navigational memory consolidation in human subjects does not rule out contributions of other sleep stages toward this phenomenon. Slow-wave sleep and its associated sharp-wave ripples have been shown to be important in spatial memory in both humans (Peigneux et al., 2004) and rodents (Girardeau et al., 2009). In fact, the sequential hypothesis (Giuditta et al., 1995) suggests that the maximal benefit of sleep on memory consolidation arises from a period of REM sleep after slow-wave sleep. The strongest correlate of overnight improvement in human visual texture discrimination was the product of the amount of slow-wave sleep in the first quarter of the night and the amount of REM in the last quarter (Stickgold et al., 2000). In any case, a contribution of REM sleep toward spatial navigational memory has important clinical implications in several sleep disorders in which REM fragmentation occurs, including not only OSA as highlighted here, but also in REM behavior disorder and narcolepsy. There may be clinical implications for individuals 
taking antidepressants known to reduce REM sleep, such as the monoamine oxidase inhibitor, SSRI, and serotonin-norepinephrine reuptake inhibitor (SNRI) classes of medicines, but these medicines have pleiotropic effects that may also act to improve memory.

Several proposals exist for the neural mechanisms by which REM sleep supports memory formation. In a model supported by the sequential hypothesis, the reactivation and redistribution of neural connections occurring during slow-wave sleep require a period of subsequent synaptic consolidation possibly afforded by subsequent REM sleep. Supporting this role for REM sleep, the waking learning of novel object information or the waking induction of hippocampal long-term potentiation (LTP) induced the REM-dependent induction of immediate early genes such as Arc and Zif-268 in primary somatosensory cortex (Ribeiro et al., 2007) and hippocampus (Ribeiro et al., 2002), respectively. REM-preferential sleep deprivation in rats resulted in impaired hippocampal LTP induction via both titanic and theta-burst stimulation protocols (McDermott et al., 2003), impaired LTP maintenance in the hippocampus, but not prefrontal cortex (Romcy-Pereira and Pavlides, 2004), and decreased hippocampal ERK activation (Ravassard et al., 2009).

Plasticity during REM sleep may be bidirectional, for example, strengthening connections encoding novel information and weakening connections encoding familiar information. This idea is supported by the observation that hippocampal place cells fired predominantly at the trough of the local theta oscillation in REM sleep after exploration of a familiar track, whereas a bimodal pattern of firing (at both peaks and troughs) was observed after exploration of a novel track (Poe et al., 2000). Along the same lines, within the hippocampus, CA1 pyramidal neuron and interneuron firing rates decrease across REM, and both these excitability changes and neuronal synchrony during sharp-wave ripples correlate with theta power in REM sleep (Grosmark et al., 2012).

In summary, our results support an important role for REM in the consolidation of spatial navigational memory in human subjects and a negative effect on this process by apnea-induced REM sleep disruption. A particular strength of this study includes the paired design, which helps control for differences in experience with $3 \mathrm{D}$ computer games. Studies using tones or other exogenous methods to disrupt REM sleep help answer scientific questions about its role. However, by using stage-specific withdrawal of CPAP to induce OSA, we not only provide mechanistic insight into the consolidation of spatial navigational memory but also describe new cognitive consequences for those left with untreated severe OSA.

\section{References}

Bjorness TE, Riley BT, Tysor MK, Poe GR (2005) REM restriction persistently alters strategy used to solve a spatial task. Learn Mem 12:352-359. CrossRef Medline

Buzsáki G, Moser EI (2013) Memory, navigation and theta rhythm in the hippocampal-entorhinal system. Nat Neurosci 16:130-138. CrossRef Medline

Diekelmann S, Born J (2010) The memory function of sleep. Nat Rev Neurosci 11:114-126. CrossRef Medline

Dinges DF, Pack F, Williams K, Gillen KA, Powell JW, Ott GE, Aptowicz C, Pack AI (1997) Cumulative sleepiness, mood disturbance, and psychomotor vigilance performance decrements during a week of sleep restricted to 4-5 hours per night. Sleep 20:267-277. Medline

Ferrara M, Iaria G, Tempesta D, Curcio G, Moroni F, Marzano C, De Gennaro L, Pacitti C (2008) Sleep to find your way: the role of sleep in the consolidation of memory for navigation in humans. Hippocampus 18 : 844-851. CrossRef Medline

Girardeau G, Benchenane K, Wiener SI, Buzsáki G, Zugaro MB (2009) Selective suppression of hippocampal ripples impairs spatial memory. Nat Neurosci 12:1222-1223. CrossRef Medline

Giuditta A, Ambrosini MV, Montagnese P, Mandile P, Cotugno M, Grassi Zucconi G, Vescia S (1995) The sequential hypothesis of the function of sleep. Behav Brain Res 69):157-166. CrossRef

Gozal D, Daniel JM, Dohanich GP (2001) Behavioral and anatomical correlates of chronic episodic hypoxia during sleep in the rat. J Neurosci 21:2442-2450. Medline

Grosmark AD, Mizuseki K, Pastalkova E, Diba K, Buzsáki G (2012) REM sleep reorganizes hippocampal excitability. Neuron 75:1001-1007. CrossRef Medline

Hagewoud R, Havekes R, Tiba PA, Novati A, Hogenelst K, Weinreder P, Van der Zee EA, Meerlo P (2010) Coping with sleep deprivation: shifts in regional brain activity and learning strategy. Sleep 33:1465-1473. Medline

Iaria G, Petrides M, Dagher A, Pike B, Bohbot VD (2003) Cognitive strategies dependent on the hippocampus and caudate nucleus in human navigation: variability and change with practice. J Neurosci 23:5945-5952. Medline

Iber C, Ancoli-Israel S, Chesson AL, Quan SF (2007) The AASM manual for the scoring of sleep and associated events: rules, terminology, and technical specifications, Ed 1. Westchester, IL: American Academy of Sleep Medicine.

Kishi A, Natelson BH, Togo F, Struzik ZR, Rapoport DM, Yamamoto Y (2011) Sleep-stage dynamics in patients with chronic fatigue syndrome with or without fibromyalgia. Sleep 34:1551-1560. Medline

Kohler M, Stoewhas AC, Ayers L, Senn O, Bloch KE, Russi EW, Stradling JR (2011) Effects of continuous positive airway pressure therapy withdrawal in patients with obstructive sleep apnea: a randomized controlled trial. Am J Respir Crit Care Med 184:1192-1199. CrossRef Medline

Kribbs NB, Pack AI, Kline LR, Getsy JE, Schuett JS, Henry JN, Maislin G, Dinges DF (1993) Effects of one night without nasal CPAP treatment on sleep and sleepiness in patients with obstructive sleep apnea. Am Rev Respir Dis 147:1162-1168. CrossRef Medline

Louie K, Wilson MA (2001) Temporally structured replay of awake hippocampal ensemble activity during rapid eye movement sleep. Neuron 29:145-156. CrossRef Medline

McDermott CM, LaHoste GJ, Chen C, Musto A, Bazan NG, Magee JC (2003) Sleep deprivation causes behavioral, synaptic, and membrane excitability alterations in hippocampal neurons. J Neurosci 23:96879695. Medline

Nguyen ND, Tucker MA, Stickgold R, Wamsley EJ (2013) Overnight sleep enhances hippocampus-dependent aspects of spatial memory. Sleep 36: 1051-1057. CrossRef Medline

Norman RG, Scott MA, Ayappa I, Walsleben JA, Rapoport DM (2006) Sleep continuity measured by survival curve analysis. Sleep 29:1625-1631. Medline

Orban P, Rauchs G, Balteau E, Degueldre C, Luxen A, Maquet P, Peigneux P (2006) Sleep after spatial learning promotes covert reorganization of brain activity. Proc Natl Acad Sci U S A 103:7124-7129. CrossRef Medline

Patel AV, Hwang D, Masdeu MJ, Chen GM, Rapoport DM, Ayappa I (2011) Predictors of response to a nasal expiratory resistor device and its potential mechanisms of action for treatment of obstructive sleep apnea. J Clin Sleep Med 7:13-22. Medline

Peigneux P, Laureys S, Fuchs S, Collette F, Perrin F, Reggers J, Phillips C, Degueldre C, Del Fiore G, Aerts J, Luxen A, Maquet P (2004) Are spatial memories strengthened in the human hippocampus during slow wave sleep? Neuron 44:535-545. CrossRef Medline

Poe GR, Nitz DA, McNaughton BL, Barnes CA (2000) Experiencedependent phase-reversal of hippocampal neuron firing during REM sleep. Brain Res 855:176-180. CrossRef Medline

Ravassard P, Pachoud B, Comte JC, Mejia-Perez C, Scoté-Blachon C, Gay N, Claustrat B, Touret M, Luppi PH, Salin PA (2009) Paradoxical (REM) sleep deprivation causes a large and rapidly reversible decrease in longterm potentiation, synaptic transmission, glutamate receptor protein levels, and ERK/MAPK activation in the dorsal hippocampus. Sleep 32: 227-240. Medline

Ribeiro S, Mello CV, Velho T, Gardner TJ, Jarvis ED, Pavlides C (2002) 
Induction of hippocampal long-term potentiation during waking leads to increased extrahippocampal zif-268 expression during ensuing rapid-eye-movement sleep. J Neurosci 22:10914-10923. Medline Ribeiro S, Shi X, Engelhard M, Zhou Y, Zhang H, Gervasoni D, Lin SC, Wada K, Lemos NA, Nicolelis MA (2007) Novel experience induces persistent sleep-dependent plasticity in the cortex but not in the hippocampus. Front Neurosci 1:43-55. CrossRef Medline

Romcy-Pereira R, Pavlides C (2004) Distinct modulatory effects of sleep on the maintenance of hippocampal and medial prefrontal cortex LTP. Eur J Neurosci 20:3453-3462. CrossRef Medline

Smith CT, Conway JM, Rose GM (1998) Brief paradoxical sleep deprivation impairs reference, but not working, memory in the radial arm maze task. Neurobiol Learn Mem 69:211-217. CrossRef Medline

Stickgold R, Whidbee D, Schirmer B, Patel V, Hobson JA (2000) Visual discrimination task improvement: a multi-step process occurring during sleep. J Cogn Neurosci 12:246-254. CrossRef Medline

Ward CP, McCoy JG, McKenna JT, Connolly NP, McCarley RW, Strecker RE (2009) Spatial learning and memory deficits following exposure to $24 \mathrm{~h}$ of sleep fragmentation or intermittent hypoxia in a rat model of obstructive sleep apnea. Brain Res 1294:128-137. CrossRef Medline

Wilson MA, McNaughton BL (1994) Reactivation of hippocampal ensemble memories during sleep. Science 265:676-679. CrossRef Medline

Yoo SS, Hu PT, Gujar N, Jolesz FA, Walker MP (2007) A deficit in the ability to form new human memories without sleep. Nat Neurosci 10:385-392. CrossRef Medline

Youngblood BD, Zhou J, Smagin GN, Ryan DH, Harris RB (1997) Sleep deprivation by the "flower pot" technique and spatial reference memory. Physiol Behav 61:249-256. CrossRef Medline 